Article

\title{
ACEnet: Approximate Thinning-Based Judicious Network Control for Energy-Efficient Ultra-Dense Networks
}

\author{
Wonseok Lee ${ }^{1}$, Bang Chul Jung ${ }^{2}$ and Howon Lee ${ }^{1,3, *(D)}$ \\ 1 Department of Electrical, Electronic, and Control Engineering and IITC, Hankyong National University, \\ Anseong, Gyeonggi 17579, Korea; longtimep@hknu.ac.kr \\ 2 Department of Electronics Engineering, Chungnam National University, Daejeon 34134, Korea; \\ bcjung@cnu.ac.kr \\ 3 Qualcomm Institute, University of California, San Diego, La Jolla, CA 92093-0021, USA \\ * Correspondence: hwlee@hknu.ac.kr or hwlee@eng.ucsd.edu; Tel.: +82-31-670-5198
}

Received: 14 April 2018; Accepted: 17 May 2018; Published: 21 May 2018

\begin{abstract}
This study considers a ultra-dense network (UDN) in which the enormous number of base stations (BSs) are densely deployed to support the massive amount of data traffic generated by many mobile devices. In this paper, we propose an approximate thinning-based judicious network control algorithm for energy-efficient UDNs (ACEnet) to improve the area throughput while diminishing the network energy consumption. The main idea of the proposed ACEnet algorithm is to judiciously adjust the modes of the BSs according to active-user density based on the thinning operation in stochastic geometry framework. The stochastic geometry framework is exploited to analyze the performance of the proposed algorithm, which includes the signal-to-interference-plus-noise ratio (SINR), average achievable rate of users, area throughput, and energy efficiency. Through intensive simulations, it shows that the proposed algorithm outperforms conventional algorithms. We also demonstrate that the analytical results are well matched with the simulation results.
\end{abstract}

Keywords: stochastic geometry; ultra-dense network; energy efficiency; hard-core point process

\section{Introduction}

In 5th generation mobile communication systems (5G), there are many promising technologies such as full-duplex radio (FDD), dynamic time division duplex (TDD), massive multiple-input multiple-output (MIMO), wireless fronthauling and backhauling, and ultra-dense networks (UDNs) to fulfill diverse system requirements [1-3]. Mobile traffic and devices are explosively increasing, and also will continually increase in the future because of many forthcoming 5G services for massive machine-type communications (mMTC) and the Internet of Things (IoT). Thus, base stations (BSs) may be more densely deployed to support the enormous number of mobile devices. However, there still exist several fundamental challenges for minimizing energy consumption, detrimental interferences, and frequent handovers in UDNs. In particular, the reduction in energy consumption is one of the most challenging technical issues for implementing UDNs in practice when considering the fact that $80 \%$ of the energy in mobile networks is consumed in BSs [4,5].

Recently, the optimization of the network energy efficiency is one of the most actively investigated research items in 5G UDNs. A scheme to adjust the BS modes (awake or sleep) was proposed [6] to minimize the energy consumption of the BSs. In [7], potential gains and limitations of the UDNs were investigated. In particular, the impact of idle-mode operation of BSs, transmission power of BSs, user density, and user distribution on the energy efficiency of the UDN is addressed. Furthermore, Reference [8] proposed a centralized power on/off optimization technique based on system-level 
simulations for heterogeneous networks. In [9], an optimal wake-up mechanism of femtocell BSs was proposed to minimize the energy consumption of heterogeneous networks by using Markov decision processes (MDP), exchanging information on the amount of data traffic between BSs and users. In addition, Reference [10] proposed energy efficient user association and power allocation methods in millimeter-wave-based ultra-dense networks with energy harvesting base stations.

A performance analysis framework based on stochastic geometry has been developed for cellular networks especially for achievable rate and coverage probability in small cells [11]. In [12,13], spectral efficiency, area throughput, and network energy efficiency of UDNs were analyzed with a stochastic geometry framework, and it is shown that a trade-off exists among such performance metrics. In addition, in [14], the BS density for optimal energy efficiency is analyzed by the stochastic geometry framework with the Newton iteration method for cellular networks where it is also shown that the network energy efficiency can be improved by carefully adjusting the BS density via switching BSs on or off according to the network traffic load. In [15], an energy-efficient resource management scheme using game theory was proposed for UDNs modeled with stochastic geometry framework where a modified K-means algorithm was utilized for the BS-clustering process. In addition, Jia et al. proposes a BS switch-off strategy with distance constraints to reduce the energy consumption in Matern hard-core point process (HCPP) random cellular networks [16]. The concept of energy balance is proposed to evaluate the efficiency of energy saving in these networks. The objective of [16] is to find a maximal point of the energy balance for the BS switch-off strategy by intensive simulations. However, because network environment and parameters are always variable, it is almost impossible to perform simulations and obtain corresponding results every time when the network conditions dynamically change.

The energy efficiency of the UDNs can be improved by appropriately adjusting the awake/sleep mode of the BS. When determining the mode of the BS, the locations of the BS is one of the most important factors when considering densely deployed BSs. Thus, we herein propose an approximate thinning-based judicious network control algorithm for energy-efficient UDNs (ACEnet). The main idea of the proposed algorithm is to decide the appropriate status of the BS considering a thinning radius $\left(r_{t}\right)$ in a well-known HCPP. In this HCPP process, determining the thinning radius is very important because it controls the number of BSs to be altered into sleep mode. Accordingly, we adaptively adjust the thinning radius according to the active user density in the network. We utilize the stochastic geometry model for the network configuration owing to the tractability of this model [11]. To validate the superiority of the proposed algorithm, numerical and simulation results are provided.

The remainder of this paper is organized as follows. In Section 2, we describe the formula of the system model using the stochastic geometry model. In Section 3, we propose the approximate thinning-based judicious network control algorithm for energy-efficient UDNs (ACEnet). In Section 4, we evaluate the performance of the conventional and proposed algorithms. In Section 5, we make conclusions.

\section{System Model}

The BSs and user equipment (UE) are generated by a homogeneous Poisson point process (PPP) with intensities $\lambda_{B}$ and $\lambda_{u}$ in the entire network, respectively, as shown in Figure 1. Figure 1 is the example of a Voronoi tessellation of the PPP. In general, this Voronoi tessellation decomposes the network space into several Voronoi cells corresponding to the homogeneous PPP with intensity $\lambda_{B}$. Homogeneous PPP generates each point independently, and thus the points could be closely positioned. Hence, the coverages of BSs could intricately overlap. The coverage overlaps may cause undesired inter-cell interferences and waste of network energy. These problems can be resolved by efficiently managing a number of the densely deployed BSs. In this paper, the thinning operation is applied to eliminate points (BSs) of HCPP according to the amount of active UE, where eliminating points implies reducing the number of active BSs. In the thinning operation, the points within the thinning radius are removed on the basis of a reference point. In Figure 1, the UE communicates with the nearest active BS. Then, the signal-to-interference-plus-noise-ratio (SINR) can be calculated using the 
desired signal strength from the nearest serving BS and the total amount of interferences from all other neighboring BSs.

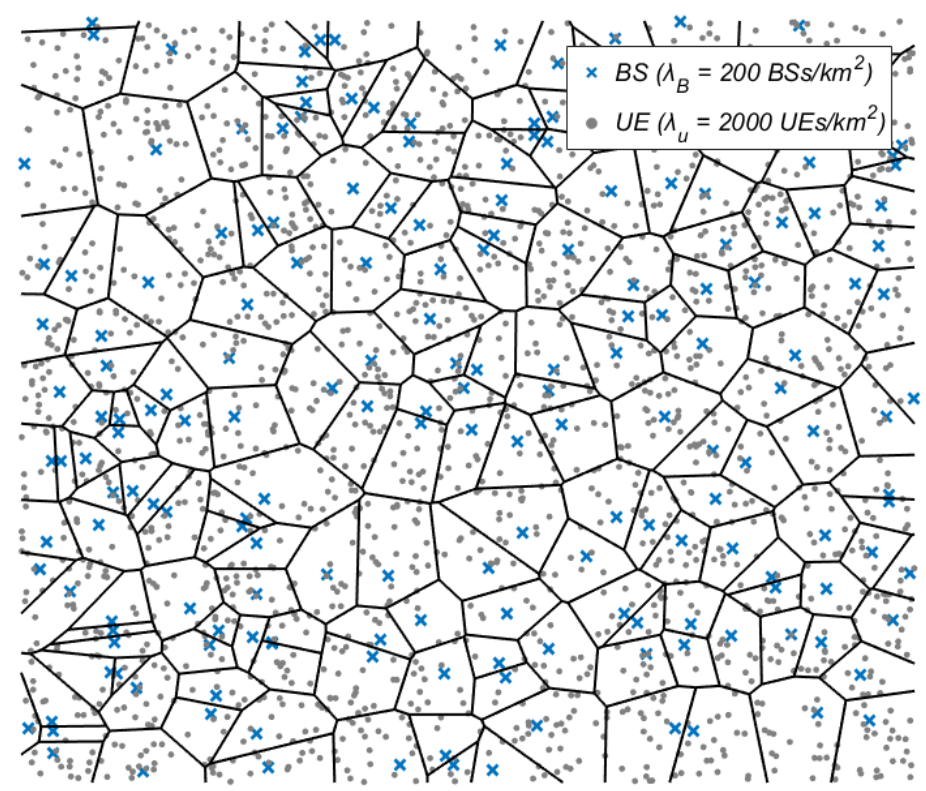

Figure 1. An example of network configuration based on stochastic geometry.

\subsection{Poisson Point Process (PPP) and Thinning Operation}

Equation (1) represents the probability that there are $n$ BSs (or UE) in a specific space S.

$$
\mathbb{P}(n)=\frac{(\lambda S)^{n}}{n !} e^{-\lambda S}
$$

Here, UE can communicate with the nearest BS and all the neighboring BSs are considered as interferers. Given that the distance between the nearest BS (serving BS) and the UE is $r$, the probability density function (pdf) of $r$ can be derived by using the fact that the null probability of the two-dimensional Poisson process defined in the specific space $S$ with a radius $R$ is $\exp (-\lambda S)$ [11]. Thus, $\mathbb{P}[r>R]$ can be calculated as

$$
\mathbb{P}[r>R]=\mathbb{P}[\text { No BS closer than } \mathrm{R}]=e^{-\lambda_{B} \pi R^{2}} .
$$

Accordingly, $\mathbb{P}[r \leq R]$ can be represented as

$$
\mathbb{P}[r \leq R]=F_{r}(R)=1-e^{-\lambda_{B} \pi R^{2}} .
$$

From Equation (3), we can obtain the pdf of $r$ in the homogeneous PPP $\left(f_{r}(r)\right)$ as

$$
f_{r}(r)=2 \pi \lambda_{B} r \cdot e^{-\lambda_{B} \pi r^{2}} .
$$

In this paper, we denote that $f_{r . p}(r)$ and $f_{r . t}(r)$ are the pdfs of $r$ in the homogeneous PPP and HCPP, respectively. As mentioned before, the thinning operation in the HCPP is the process to create a group of new points by removing the points within the thinning radius $\left(r_{t}\right)$ on the basis of reference points among all the original points generated by the homogeneous PPP. In this paper, the thinning operation removes points according to the following steps:

- $\quad$ Step 1: Assign a random marked value (M) between 0 and 1 for all points sampled by PPP. 
- Step 2: Find the points within the thinning radius on the basis of the reference point, and remove the points with a smaller value than that of the reference point.

- $\quad$ Step 3: Repeat Step 1 and Step 2 for all points in the entire network.

Accordingly, the intensity of the BS in the network is altered and the pdf of the distance $r$ between the BS and the UE is also changed. In this case, the pdf of $r$ after applying the thinning operation can be approximated as $[16,17]$

$$
f_{r . t}(r) \approx 2 \pi r \lambda_{\text {B.p }} \frac{1-e^{-\lambda_{\text {B.p }} \pi r_{t}^{2}}}{\lambda_{\text {B.p }} \pi r_{t}^{2}} e^{-\lambda^{s} A\left(r, r_{t}\right)},
$$

where $\lambda_{\text {B.p }}$ represents the BS intensity of original PPP, and the intensity of the remained BSs after the thinning operation can be expressed as $\lambda_{B . t}=\left(1-e^{-\lambda_{B . p} \pi r_{t}^{2}}\right) / \pi r_{t}^{2}$ [18-20]. In Equation (5), $\lambda^{s}$ is a unique value that matches the sum of the pdf to 1 . In addition, $A\left(r, r_{t}\right)$ in Equation (5) can be described as follows [16]:

$$
\mathrm{A}\left(r, r_{t}\right)= \begin{cases}0, & 2 r \leqq r_{t} \\ \pi r^{2}+r_{t} \sqrt{r^{2}-\frac{r_{t}^{2}}{4}}-\left(2 \cdot \arcsin \frac{r_{t}}{2 r}+\arccos \frac{r_{t}}{2 r}\right) \times r^{2}, & 2 r>r_{t} .\end{cases}
$$

$N\left(r_{i}\right)$ is the average amount of UE within the $i$ th integration region. The entire space $\mathrm{S}$ in Equation (1) can be quantized into bins with bounds $\left(r_{i}-\Delta r / 2, r_{i}+\Delta r / 2\right)$, where $r_{i}=r_{0}+i \cdot \Delta r$ for $i \in \mathbb{Z}^{+}$[12]. Here, we assume that $r_{0}$ is a clearance region, where no UE is located. Therefore, $N\left(r_{i}\right)$ of the $i$ th integration region whose area is $2 \pi r_{i} \Delta_{r}$ can be expressed as [12,13]

$$
N\left(r_{i}\right)=\sum_{k} k \cdot \frac{\left(\lambda_{u} 2 \pi r_{i} \Delta r\right)^{k}}{k !} e^{-\lambda_{u} 2 \pi r_{i} \Delta r}, \quad k \in \mathbb{Z}^{+} .
$$

From Equations (4) and (6), we can calculate the total amount of UE within the BS coverage. Therefore, the amount of UE in the BS coverage $\left(N_{u . t o t}\right)$ is approximately expressed as

$$
N_{\text {u.tot }}=\sum_{i} p_{r}\left(r_{i}\right) \cdot N\left(r_{i}\right)
$$

where the probability mass function $p_{r}\left(r_{i}\right)$ can be calculated as $p_{r}\left(r_{i}\right)=\int_{r_{i}-\Delta r}^{r_{i}+\Delta r} f_{r}(r) d r$. In addition, in accordance with [21], the average amount of UE in each BS $\left(N_{u . t o t}\right)$ can be expressed as $\frac{\lambda_{u}}{\lambda_{B}}$.

\subsection{Average Achievable Rate, Average Cell Throughput, and Energy Efficiency}

The instantaneous SINR of typical UE whose distance from the BS is $r$ can be expressed as

$$
\operatorname{SINR}(\mathrm{r})=\frac{P_{t} h r^{-\alpha}}{I_{r}+N_{0}}
$$

where $P_{t}, h$, and $\alpha$ denote the transmission power of the BS, small-scale fading (e.g., Rayleigh fading), and a path-loss exponent, respectively. In addition, $N_{0}$ is noise power and assumed to be additive with variance $\left(\sigma^{2}\right)$. In Equation (8), the cumulative interference from all the other BSs except its associated BS $\left(I_{r}\right)$ can be calculated as

$$
I_{r}=\sum_{i \in \mathbb{B} / b_{0}} P_{t} h v_{i}^{-\alpha},
$$

where $\mathbb{B}$ is the set of all the BSs in the entire network, and $b_{0}$ denotes the UE's associated BS. In addition, $v_{i}$ is the distance from neighbor BSs that cause the interferences. From Shannon formula, the instantaneous spectral efficiency of a typical UE of distance $r$ from its associated BS is 


$$
R(r)=\log _{2}(1+\operatorname{SINR}(\mathrm{r}))=\log _{2}\left(1+\frac{P_{t} h r^{-\alpha}}{I_{r}+N_{0}}\right) .
$$

From Equation (10), the average ergodic rate $\left(\mathbb{E}_{h, I_{r}}[R(r)]\right)$ can be calculated as $[11,22]$

$$
\begin{aligned}
\mathbb{E}_{h, I_{r}}[R(r)] & =\mathbb{E}_{I_{r}}\left[\mathbb{E}_{h}[\ln (1+\operatorname{SINR}(\mathrm{r}))]\right] \\
& =\mathbb{E}_{I_{r}}\left[\int_{t>0} \mathbb{P}\left[\ln \left(1+\frac{P_{h} h r^{-\alpha}}{I_{r}+N_{0}}\right)>t\right] d t\right] \\
& =\mathbb{E}_{I_{r}}\left[\int_{t>0} \mathbb{P}\left[h>\mu r^{\alpha}\left(e^{t}-1\right)\left(I_{r}+N_{0}\right)\right] d t\right] \\
& =\int_{t>0} e^{-\mu r^{\alpha}\left(e^{t}-1\right) N_{0}} \cdot \mathbb{E}_{I_{r}}\left[e^{-\mu r^{\alpha}\left(e^{t}-1\right) I}\right] d t \\
& =\int_{t>0} e^{-\mu r^{\alpha}\left(e^{t}-1\right) N_{0}} \cdot \mathcal{L}_{I_{r}}\left(-\mu r^{\alpha}\left(e^{t}-1\right)\right) d t .
\end{aligned}
$$

Here, we assume a Rayleigh fading channel and $\mu=1 / P_{t}$. In Equation (11), $\mathcal{L}$ denotes a Laplace transform and $-\mu r^{-\alpha}\left(e^{t}-1\right)$ can be replaced by $s$. Thus, the Laplace transform of $I_{r}\left(\mathcal{L}_{I_{r}}(s)\right)$ can be obtained as

$$
\begin{aligned}
\mathcal{L}_{I_{r}}(s) & =\mathbb{E}_{I_{r}}\left[e^{-s I_{r}}\right] \\
& =\mathbb{E}_{\mathbb{B}, h_{i}}\left[\exp \left(-s \sum_{i \in \mathbb{B} / b_{0}} P_{t} h_{i} v_{i}^{-\alpha}\right)\right] \\
& =\mathbb{E}_{\mathbb{B}}\left[\prod_{i \in \mathbb{B} / b_{0}} \mathbb{E}_{h}\left[e^{-s P_{t} h v_{i}^{-\alpha}}\right]\right] \\
& =e^{-2 \pi \lambda \int_{r}^{\infty}\left(1-\mathbb{E}_{h}\left[\exp \left(-s P_{t} h v^{-\alpha}\right)\right]\right) v d v} \\
& =e^{-2 \pi \lambda \int_{r}^{\infty}\left(1-\left(\int_{0}^{\infty} \exp \left(-s h v^{-\alpha}\right) \exp (-h) d h\right) v d v\right.} \\
& =e^{-2 \pi \lambda \int_{r}^{\infty}\left(1-\frac{\mu}{s v^{-\alpha}+\mu}\right) v d v}
\end{aligned}
$$

Then, from Equations (4), (5), and (11), the average achievable rate is expressed as [12,13]

$$
T=\int_{r} f_{r}(r) \cdot B_{u} \cdot \mathbb{E}_{h, I_{r}}[R(r)] d r,
$$

where $B_{u}$ is the size of bandwidth allocated to each UE. For the sake of simplicity, we assume that the total bandwidth $(B)$ is equally allocated to all the users $\left(N_{u}\right)$ in this paper. When the BSs and UE are deployed by original PPP without the thinning operation, the average achievable rate can be obtained by Equations (4), (11), and (13). However, when the BSs are deployed by PPP with thinning operation, the average achievable rate can be calculated by Equations (5), (11), and (13).

In [19], the tight bound of the received interference power is proposed by setting the UE's position as the same with the tagged BS's position. The interfering signals are generated at BSs farther than the thinning radius, and the results are obtained against thinning radius. The difference of the received interference power between HCPP and PPP is around 1-2 dB. Figure 2 shows the received interference power after the thinning operations in the HCPP and PPP. These results are the normalized interference power divided by the BS intensity. The square symbols represent the HCPP with the BS intensity $\left(\lambda_{B . t}\right)$, and the circles indicate the PPP with the BS intensity $\left(\lambda_{B}\right)$.

In addition, the average cell throughput is derived from Equations (7) and (13). In other words, the average cell throughput can be calculated by $N_{u . t o t} \cdot T$, where there is $N_{u \text {.tot }}$ UE in the BS and their average achievable rate can be obtained by Equation (13). Therefore, the area throughput $\left(T_{\text {area }}\right)$ per $\mathrm{km}^{2}$ can be obtained by multiplying the average cell throughput by $\lambda_{B}$ :

$$
T_{\text {area }}=\lambda_{B} \times N_{u \text {.tot }} \times T=\lambda_{B} \times N_{u \text {.tot }} \times \int_{r} f_{r}(r) \cdot B_{u} \cdot \mathbb{E}_{h, I_{r}}[R(r)] d r .
$$


In addition, from Equation (14), the energy efficiency $\left(\eta_{\text {area }}\right)$ in the network is represented as

$$
\eta_{\text {area }}=\frac{T_{\text {area }}}{\lambda_{B} \times P_{B}}=\frac{T_{\text {area }}}{\lambda_{B} \times\left(\frac{1}{\sigma} P_{t}+P_{c}+P_{0}\right)} .
$$

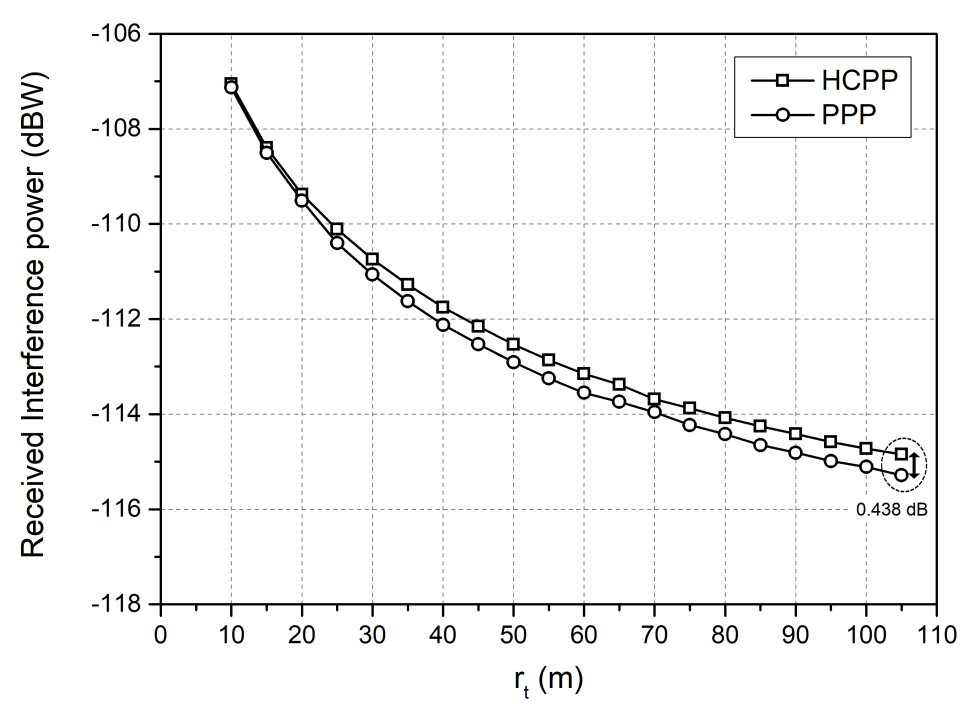

Figure 2. Received interference power by PPP and HCPP vs. thinning radius $\left(r_{t}\right)$.

Here, $P_{B}$ is the total energy consumed by the BS. It can be calculated as $P_{B}=(1 / \sigma) \cdot P_{t}+P_{c}+P_{0}$ where $\sigma$ is the amplification efficiency, and $P_{\mathcal{C}}$ and $P_{0}$ are the consumed power by the circuit and in the standby mode, respectively.

\section{ACEnet: Proposed Approximate Thinning Based Judicious Network Control Algorithm}

In this section, we describe how to decide the appropriate status of the BSs to improve energy efficiency in the UDNs. Algorithm 1 describes the overall procedures of the proposed approximate thinning-based judicious network control algorithm with thinning operation (ACEnet). This algorithm represents a process to find the thinning radius $r_{t}$. Here, $N_{B}$ and $N_{u}$ denote the number of BSs and the amount of UE, respectively, $C_{B}$ represents the amount of UE that can be accommodated at once in the $\mathrm{BS}$, and $d_{i, j}$ means the inter-site distance between BS $i$ and BS $j . M_{i}$ denotes a unique random mark value between 0 and 1 for performing the thinning operation. For BS $k$ randomly chosen among the set of all BSs (B), we create the BS group $G_{I N}^{[k]}$. By comparing $M_{i}$ in $G_{I N}^{[k]}$ with $M_{k}$, we can determine the BS whose mode will be changed into sleep. Algorithm 1 consists of two parts as follows.

- Part 1-Calculation of $r_{t}$ : By using $N_{u}$ and $C_{B}$, we can calculate approximately how many BSs must be awake to serve active UE, where the number of BSs to be awake is $N_{R}$. Accordingly, the number of BSs to be in sleep mode is calculated as $N_{B}-N_{R}$, and the ratio of BSs in sleep mode to BSs in awake mode is calculated by $\tilde{N}_{r}=\left(N_{B}-N_{R}\right) / N_{R}$. Consequently, the thinning radius that satisfies $F_{d_{i, j}}\left(r_{t}\right)=P_{r}$ can be obtained from the cumulative distribution function $(\mathrm{CDF})$ of the inter-site distance between BSs $\left(d_{i, j}\right)$, where $P_{r}$ is the ratio of $\widetilde{N}_{r}$ to $N_{B}$.

- Part 2-Calculation of $\Psi$ : A matrix $\Psi$ represents the overall status of all BSs. The BSs within the thinning radius are classified as Group $G_{I N}^{[k]}$ according to the distance $\left(d_{k, j}\right)$ between the BS $k$ and its neighboring BSs. After comparing $M_{i}$ included in $G_{I N}^{[k]}$, the mode of the BS that satisfies $M_{i, i \neq k}<M_{k}$ is changed to sleep mode. As shown in Algorithm 1, this procedure is conducted iteratively for all BSs. 


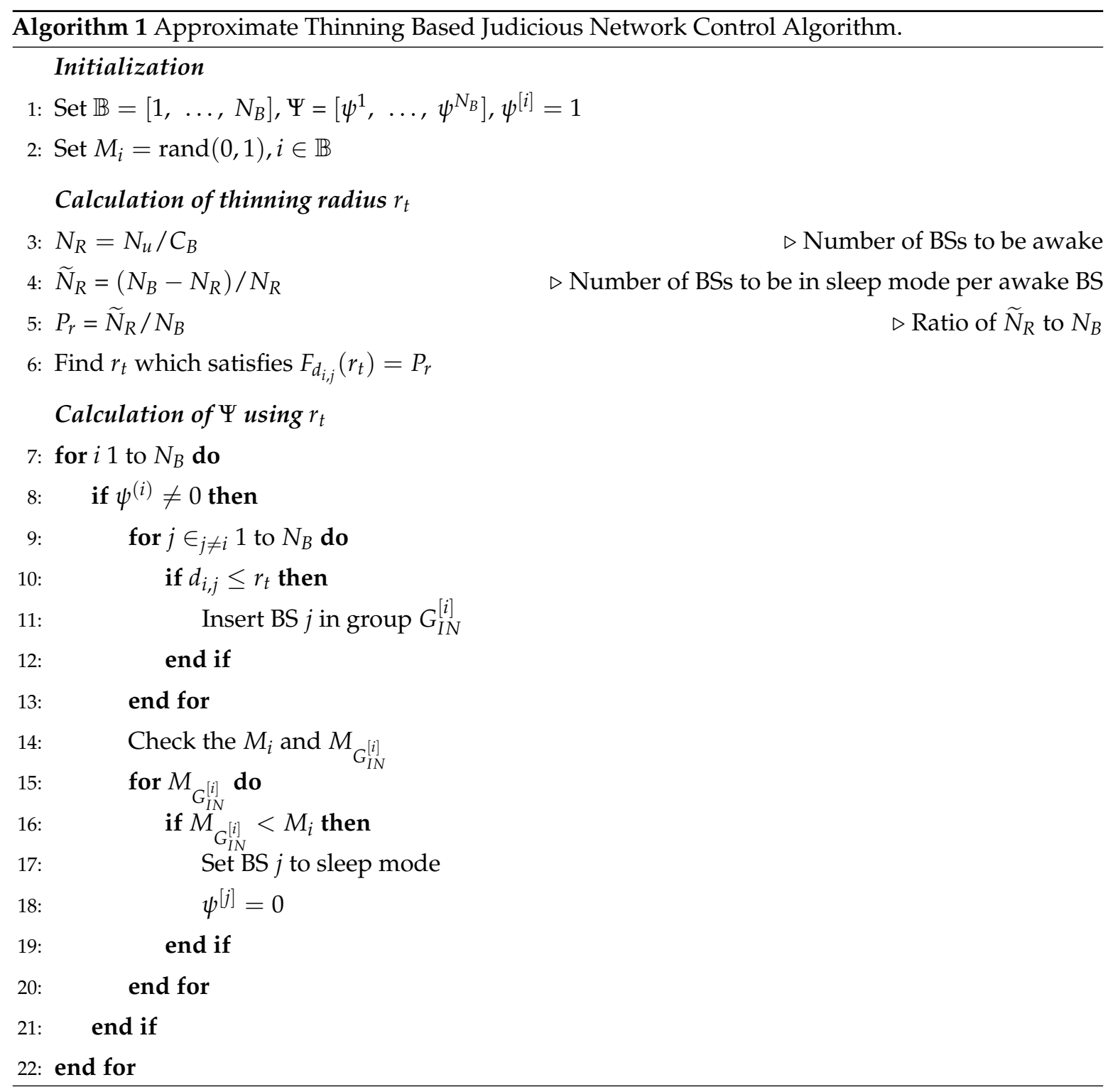

In summary, the BS to be awake and the BS to be in sleep mode can be determined through Algorithm 1, and the results are updated in $\Psi$. Namely, the updated $\Psi$ indicates whether the BS should be awake or in sleep mode. Figure 3 shows the example of judicious BS control based on the proposed approximate thinning operation.

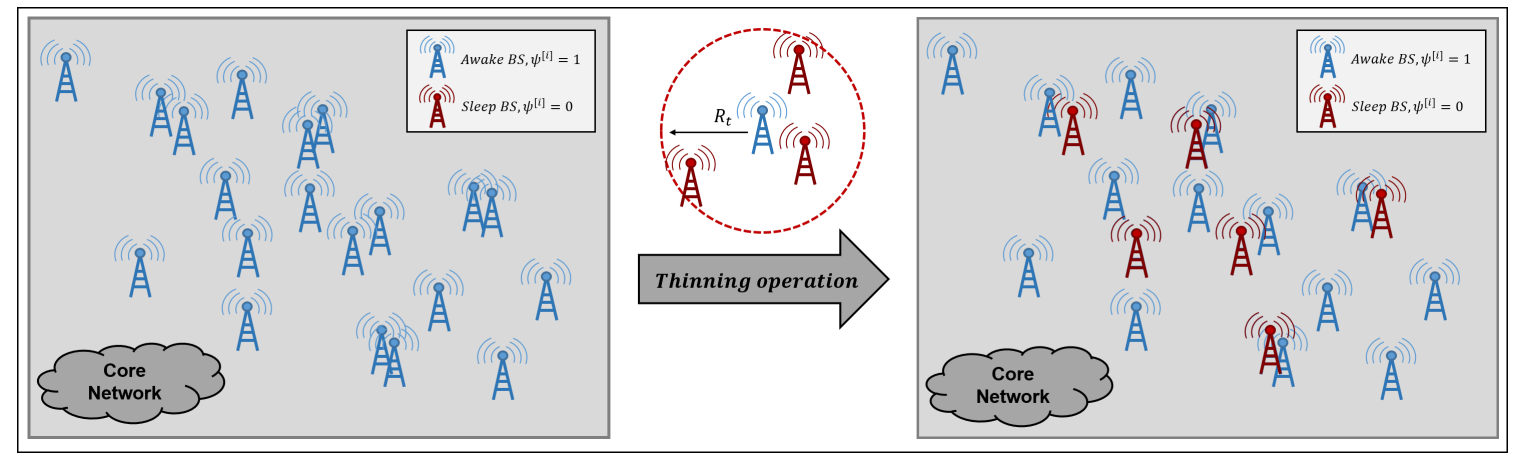

Figure 3. Example of judicious BS control based on approximate thinning operation. 


\section{Numerical and Simulation Results}

In this paper, to improve the energy efficiency of the UDNs, we proposed the ACEnet algorithm based on the thinning operation. In our proposed algorithm, the status of the BSs is adaptively changed according to the amount of active UE in the networks. We herein analyze and discuss the performance of our proposed algorithm according to the variation in amount of UE. The amount of UE increases from 1000 to 120,000, and the number of BSs is 500. The simulation parameters are shown in Table 1.

Table 1. Simulation parameters.

\begin{tabular}{cc}
\hline Parameter & Value \\
\hline Network Size & $1000 \mathrm{~m} \times 1000 \mathrm{~m}$ \\
Total Bandwidth $(B)$ & $10 \mathrm{MHz}$ \\
Thermal Noise Density $\left(N_{0}\right)$ & $-120 \mathrm{dBm} / \mathrm{Hz}$ \\
User density $\left(\lambda_{u}\right)$ & $2000,1000 \sim 120,000 / \mathrm{km}^{2}$ \\
BS density $\left(\lambda_{B}\right)$ & $15 \sim 50,500 / \mathrm{km}^{2}$ \\
Path-loss Exponent $(\alpha)$ & 3.5 \\
BS Transmission Power $\left(P_{t}\right)$ & $0.25 \mathrm{~W}$ \\
BS Capacity $\left(C_{B}\right)$ & 100 \\
Amplification Efficiency $(\sigma)$ & 0.23 \\
\hline
\end{tabular}

To compare the performance results with respect to area throughput and energy efficiency, we compare our proposed ACEnet algorithm with the always-awake (AA), association-control (AC), and exhaustive search (ES) algorithms. In the AA algorithm, because the thinning operation is not utilized, all BSs are always kept in awake mode. The AC algorithm adjusts the status of BS according to the threshold number of users $\left(N_{t h}\right)$ [23]. That is, the BSs having over the threshold number of users become awake. In addition, the ES algorithm shows the upper bound of the energy efficiency in UDNs; in other words, ES finds the optimal thinning radius to achieve the best energy efficiency from 0 to $r_{\max }$, where $r_{\max }$ is the maximum thinning radius. In addition, we compare the numerical results of the average achievable rate in Equation (13) with the simulation results. In Figures 4 and 5, S and N indicate the simulation results and the numerical results, respectively.

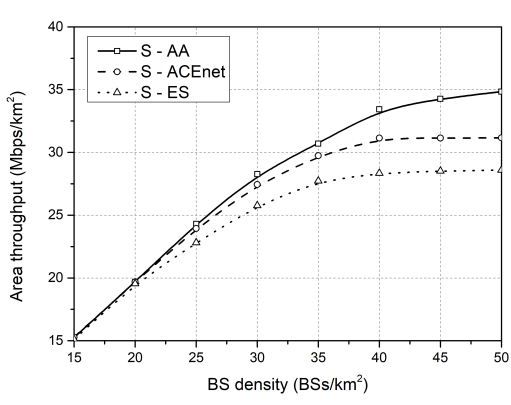

(a) Area Throughput

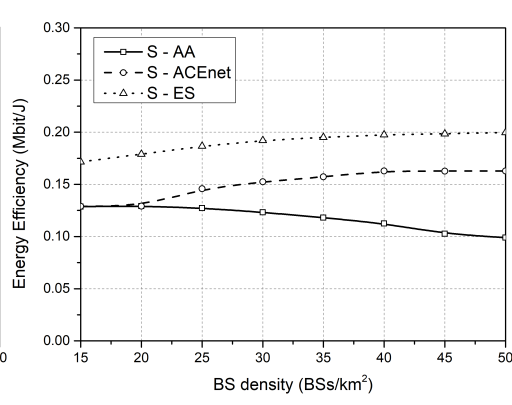

(b) Energy Efficiency

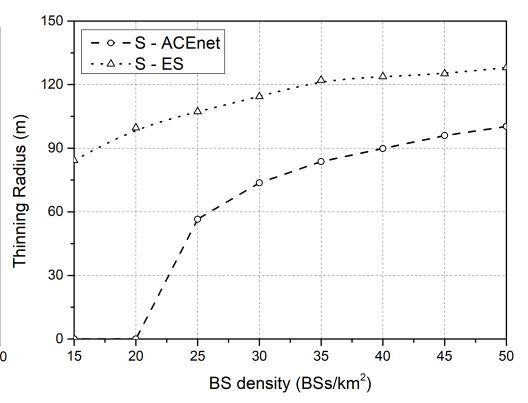

(c) Thinning Radius

Figure 4. Area throughput, energy efficiency, and thinning radius: ACEnet vs. ES. 


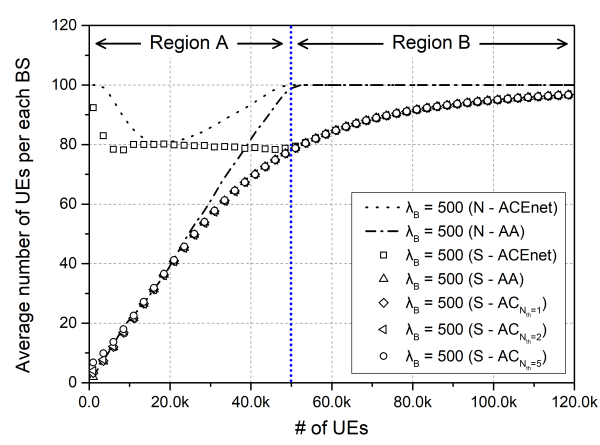

(a) \# of UE per BS

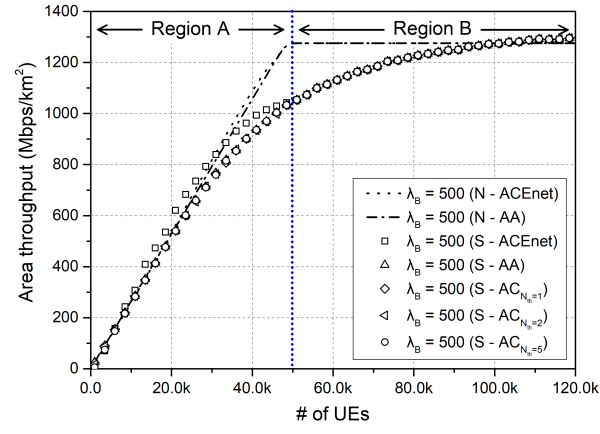

(b) Area Throughput

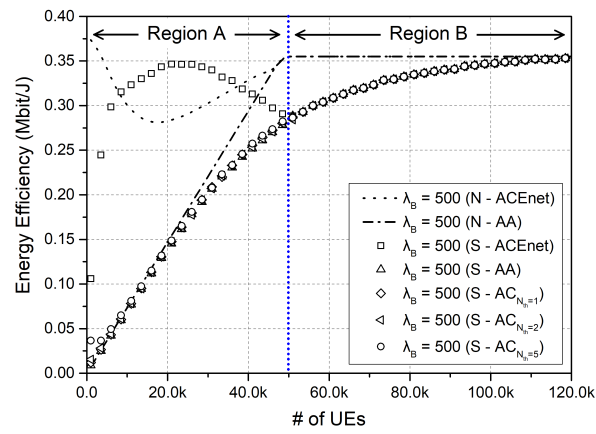

(c) Energy Efficiency

Figure 5. Average amount of UE per BS, area throughput, and energy efficiency: ACEnet vs. AA vs. AC.

\subsection{Performance Comparison with Exhaustive Search (ES) Algorithm}

The ES algorithm shows the upper bound of energy efficiency. The ES algorithm finds the optimal thinning radius to achieve the optimal energy efficiency from 0 to $r_{\max }$. Here, the number of BSs varies from 15 to 50 , and the amount of UE is 2000 . Because of the excessively long simulation time of the ES algorithm, we used a small number of BSs to obtain the simulation results.

As shown in Figure 4a, the AA algorithm has the highest area throughput among these algorithms because the status of all BSs is maintained as awake. However, because the thinning operation is applied considering the number of active UE in the proposed ACEnet algorithm, the area throughput could not exceed the area throughput in the AA algorithm.

However, as shown in Figure 4b, we can see that the energy efficiency of the proposed ACEnet algorithm is higher than that of the AA algorithm by reducing the number of awake BSs. Although the area throughput of the proposed ACEnet algorithm is slightly smaller than that of the AA algorithm, the energy efficiency is higher.

In Figure 4c, the thinning radius in the ES algorithm is larger than that of the ACEnet algorithm. This means that more BSs could enter sleep mode in the ES algorithm compared with the proposed ACEnet algorithm. This is because the goal of the ES algorithm is to maximize the energy efficiency. Furthermore, we do not need to define the thinning radius in the AA algorithm, as the thinning operation does not exist. In the ACEnet algorithm, $r_{t}$ is 0 when $\lambda_{B}$ is less than 20 . This means that the number of BSs is less than $N_{R}$. However, even in this region, the thinning operations are still applied in the ES algorithm for maximizing the energy efficiency of UDNs. From these simulation results, we can find that precise information regarding the location of active UE is very critical to determine which BSs should be set to sleep mode. 


\subsection{Performance Comparison in UDNs}

The average amount of UE per BS, the area throughput, and the energy efficiency in the UDNs are shown in Figure 5a-c, respectively. When the amount of UE is from 1000 to 50,000, $N_{R}<N_{B}$, and past the vertical line, $N_{R} \geq N_{B}$. This means that the proposed ACEnet algorithm is not conducted in region $\mathrm{B}$, when $N_{R} \geq N_{B}$. As shown in Figure 5a, in region $\mathrm{A}$, the average number of users per BS in the numerical results are greater than that in the simulation results because the number of awake BSs in the simulation results is larger than $N_{R}$ calculated by the numerical analysis. In order words, the thinning radius derived by the proposed ACEnet algorithm may have a slight difference with the numerical $r_{t}$ obtained by $F_{d_{i, j}}\left(r_{t}\right)=P_{r}$. Therefore, the amount of UE included in each BS may be reduced because of the relatively higher number of awake BSs.

In region $\mathrm{B}$, the status of all BSs is awake because $N_{R} \geq N_{B}$. Here, the gaps are caused by the non-uniformly distributed UE in the network. That is, the amount of UE per BS in simulations is not exactly even. Accordingly, the amount of interference occurred from each BS can be slightly different. However, the amount of interference in numerical results is calculated by the multiplication of the average value. For this reason, there is a difference between the simulation and numeral results, as shown in Figure $5 b, c$.

In Figure 5b, unlike the results in Section 4.1, the proposed ACEnet algorithm has a larger area throughput than the AA and AC algorithms because the amount of interference generated by the awake BSs is relatively small. For the same reason, in Figure $5 c$, we find that the proposed ACEnet algorithm has higher energy efficiency compared to those of the AA and AC algorithms when the number of iterations between 0 and the vertical line.

\section{Conclusions}

In this paper, we proposed the approximate thinning-based judicious network control to improve the energy efficiency of UDNs. The area throughput in UDNs can be increased by the judicious adjustment of the mode of a BS according to the active user density. In our proposed algorithm, the thinning radius is obtained from the amount of UE when the total number of awake BSs is less than the total number of BSs. As shown in the simulation results, the proposed ACEnet algorithm can increase energy efficiency while minimizing the decrement of the area throughput compared with the AA and AC algorithms. With our proposed ACEnet algorithm, the network operators can effectively manage the BSs in accordance with the amount of active UE to enhance the network energy efficiency.

Acknowledgments: This research was supported in part by Basic Science Research Program through the National Research Foundation of Korea (NRF) funded by the Ministry of Education (NRF-2016R1D1A1B03935902) and in part by the National GNSS Research Center program of Defense Acquisition Program Administration and Agency for Defense Development.

Author Contributions: All the authors contributed substantially to all aspects of this article; Conceptualization, H.L. and B.C.J.; Performance Analysis \& Simulation, W.L. and H.L.; Writing-Original Draft Preparation, W.L. and H.L.; Writing-Review \& Editing, H.L. and B.C.J.; Supervision, H.L.; Project Administration, H.L. and B.C.J.

Conflicts of Interest: The authors declare no conflict of interest.

\section{References}

1. Recommendation ITU-R M-2083-0. IMT Vision-Framework and Overall Objectives of the Future Development of IMT for 2020 and Beyond; ITU-R WP5D; Electronic Publication: Geneva, Switzerland, 2015.

2. Andrews, J.G.; Buzzi, S.; Choi, W.; Hanly, S.V.; Lozano, A.; Soong, A.C.K.; Zhang, J.C. What will 5G be? IEEE J. Sel. Areas Commun. 2014, 32, 1065-1082. [CrossRef]

3. Queseth, O.; Aziz, D.; Kusume, K.; Tullberg, H.; Fallgren, M.; Schellmann, M.; Uusitalo, M.; Maternia, M. ICT-317669-METIS/D8.4 V1 METIS Final Project Report. Available online: https:/ /www.metis2020.com/ wp-content/uploads/deliverables/METIS_D8.4_v1.pdf (accessed on 30 April 2015). 
4. Tombaz, S.; Usman, M.; Zander, J. Energy efficiency improvements through heterogeneous networks in diverse traffic distribution scenarios. In Proceedings of the 6th International ICST Conference on Communications and Networking in China (CHINACOM), Harbin, China, 17-19 August 2011.

5. Richter, F.; Fehske, A.J.; Fettweis, G.P. Energy efficiency aspects of base station deployment strategies for cellular networks. In Proceedings of the IEEE 70th Vehicular Technology Conference Fall (VTC 2009-Fall), Anchorage, AK, USA, 20-23 September 2009.

6. Hasan, Z.; Boostanimehr, H.; Bhargava, V.K. Green cellular networks: A survey, some research issues and challenges. IEEE Commun. Surv. Tutor. 2011, 13, 524-540. [CrossRef]

7. Lopez-Perez, D.; Ding, M.; Claussen, H.; Jafari, A.H. Towards 1 Gbps/UE in cellular systems: Understanding ultra-dense small cell deployments. IEEE Commun. Surv. Tutor. 2015, 17, 2078-2101. [CrossRef]

8. Koudouridis, G.P.; Gao, H.; Legg, P. A centralised approach to power on-off optimisation for heterogeneous networks. In Proceedings of the Vehicular Technology Conference (VTC Fall), Quebec City, QC, Canada, 3-6 September 2012.

9. Saker, L.; Elayoubi, S.-E.; Combes, R.; Chahed, T. Optimal control of wake up mechanisms of femtocells in heterogeneous Networks. IEEE J. Sel. Areas Commun. 2012, 30, 664-672. [CrossRef]

10. Zhang, H.; Huang, S.; Jiang, C.; Long, K.; Leung, V.C.; Poor, H.V. Energy Efficient User Association and Power Allocation in Millimeter-Wave-Based Ultra Dense Networks With Energy Harvesting Base Stations. IEEE J. Sel. Areas Commun. 2017, 35, 1936-1947. [CrossRef]

11. Andrews, J.G.; Baccelli, F.; Ganti, R.K. A tractable approach to coverage and rate in cellular networks. IEEE Trans. Commun. 2011, 59, 3122-3134. [CrossRef]

12. Li, Q.; Wu, G.; Hu, Q. Analytical Study on Network Spectrum Efficiency of Ultra Dense Networks. In Proceedings of the 2013 IEEE 24th International Symposium on Personal Indoor and Mobile Radio Communications (PIMRC), London, UK, 8-11 September 2013; pp. 2764-2768.

13. Ren, Q.; Fan, J.; Luo, X.; Xu, Z.; Chen, Y. Analysis of spectral and energy efficiency in ultra-dense network. In Proceedings of the 2015 IEEE International Conference on Communication Workshop (ICCW), London, UK, 8-12 June 2015.

14. An, L.; Zhang, T.; Feng, C. Stochastic geometry based energy-efficient base station density optimization in cellular networks. In Proceedings of the IEEE International Conference on Communication Workshop (ICCW), London, UK, 8-12 June 2015.

15. Liang, L.; Wang, W.; Fu, S. A cluster-based energy-efficient resource management scheme for ultra-dense networks. IEEE Access 2016, 4, 6823-6832. [CrossRef]

16. Jia, H.; Chen, J.; Ge, X. Switch-off strategy of base stations in HCPP random cellular networks. In Proceedings of the 2016 IEEE International Conference on Communications (ICC), Kuala Lumpur, Malaysia, 22-27 May 2016.

17. Alfano, G.; Garetto, M.; Leonardi, E. New insights into the stochastic geometry analysis of dense CSMA networks. In Proceedings of the IEEE INFOCOM, Shanghai, China, 10-15 April 2011; pp. 2642-2650.

18. Matern, B. Spatial Variation, 2nd ed.; Springer Lecture Notes in Statistics; Springer Science \& Business Media: Berlin, Germany, 1986.

19. Haenggi, M. Mean Interference in Hard-Core Wireless Networks. IEEE Commun. Lett. 2011, 15, 792-794. [CrossRef]

20. Dhillon, H.S.; Ganti, R.K.; Baccelli, F.; Andrews, J.G. Modeling and analysis of k-tier downlink heterogeneous cellular networks. IEEE J. Sel. Areas Commun. 2012, 30, 550-560. [CrossRef]

21. Foss, S.G.; Zuyev, S.A. On a Voronoi aggregative process related to a bivariate Poisson process. Adv. Appl. Probab. 1996, 28, 965-981. [CrossRef]

22. ElSawy, H.; Hossain, E.; Haenggi, M. Stochastic geometry for modeling, analysis, and design of multi-tier and cognitive cellular wireless networks: A survey. IEEE Commun. Surv. Tutor. 2013, 15, 996-1019. [CrossRef]

23. Jin, H.; Wu, X.; Kim, H.-S.; Jung, B.C. Energy Efficiency of Ultra-Dense Small-Cell Networks with Adaptive Cell-Breathing. IET Commun. 2018, 12, 367-372. [CrossRef]

(C) 2018 by the authors. Licensee MDPI, Basel, Switzerland. This article is an open access article distributed under the terms and conditions of the Creative Commons Attribution (CC BY) license (http://creativecommons.org/licenses/by/4.0/). 